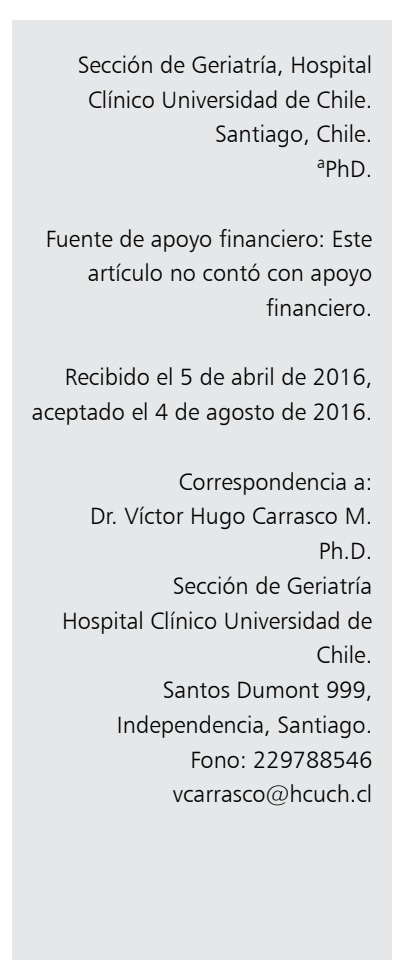

\section{Eutanasia en Chile: una discusión pendiente}

\author{
VÍCTOR HUGO CARRASCO M. ${ }^{a}$, FRANCISCA CRISPI
}

\section{Euthanasia in Chile}

\begin{abstract}
Euthanasia is a complex medical procedure. Even though end of life decisions are common situations in health practice, there is a lack of consensus about their terminology. In this manuscript, the main concepts about this issue are defined and delimited; including active and passive euthanasia and limitation of therapeutic effort. Then, a revision is made about the international experience on euthanasia, to then go through the Chile's history in euthanasia and the population's opinion. In Chile, euthanasia is an act that has been removed from the social dialogue and legislation. In order to have an open discussion in our population about the issue, the debate has to be opened to the citizens, accompanied by clear medical information about the procedure.
\end{abstract}

(Rev Med Chile 2016; 144: 1598-1604)

Key words: Ageing; Biomedical Ethics; Death with Dignity; Euthanasia; Suicide, Assisted.

\section{E} $\mathrm{n}$ abril de 2005, dos reconocidos hombres de 83 años fallecían de enfermedades terminales. Uno de ellos decidió limitar los esfuerzos y quedarse en su cama, y partió acompañado de sus seres cercanos a los pocos días. El segundo, estuvo casi 3 meses en la UCI y murió una madrugada sin su familia. El primero se llamaba Karol Wojtyla y el segundo Rainero de Mónaco.

¿Podemos decidir cómo morir? ¿Podemos intervenir en el proceso de nuestra propia muerte?

Con el aumento en la esperanza de vida a nivel mundial, aumenta también la complejidad de la atención de enfermos terminales y los dilemas éticos relacionados. La Eutanasia, del griego eu-tanatos, cuyo significado etimológico es buen morir, es uno de los grandes temas de la bioética al que se tienen que enfrentar los médicos en su práctica clínica cotidiana. Sin embargo, es un tópico que hasta hoy permanece alejado del debate público.

A pesar de que el debate sobre el final de la vida se tiende a tildar de contemporáneo, debemos recordar que históricamente las distintas culturas se han enfrentado al final de la vida de formas diversas. Las concepciones sobre la muerte, inician con el desarrollo de la conciencia del Homo Sapiens ${ }^{1}$. En la cultura griega y romana, el suicidio se aceptaba como práctica. Posteriormente, en el año 500 AC, Hipócrates en su Juramento señala "A nadie daré una droga mortal aun cuando me sea solicitada, ni daré consejo con este fin" ${ }^{2}$. Luego, vemos cómo la cultura judeo-cristiana se ha opuesto al derecho a la muerte ${ }^{2}$, argumentando que nadie puede terminar con la vida de un ser humano, ya que se violaría la ley divina ${ }^{3}$. Por otro lado, la cultura oriental se ha acercado a la muerte desde una concepción positiva, por permitirle al hombre salir de los ciclos de reencarnación ${ }^{4}$. En esta cultura, si bien aún no tienen legislación al respecto, las creencias religiosas no se oponen explícitamente al derecho a morir ${ }^{5}$.

Existe una falta de consenso con respecto a la terminología sobre la eutanasia, que dificulta el diálogo transparente y claro de la sociedad civil y la comunidad médica. A continuación, se plantean como se entenderán por el artículo algunos conceptos clave para iniciar la discusión. 


\section{Aproximaciones conceptuales}

\section{Eutanasia}

La Organización Mundial de la Salud la define como la acción deliberada que realiza una persona con la intención de provocar la muerte sin dolor, a otro sujeto, o no prevenir la muerte por causa natural, en caso de enfermedad terminal o coma irreversible. El paciente debe estar cursando con un sufrimiento físico, emocional o espiritual incontrolable, siendo el objetivo de la eutanasia aliviar este sufrimiento. Ejemplos de estos estados terminales pueden ser; cáncer en etapa de cuidados paliativos, demencia avanzada, insuficiencias severas de órganos vitales, disnea severa refractaria a tratamiento, entre otros.

La literatura ha definido como enfermo terminal a quien padece una condición patológica grave, de carácter progresivo e irreversible que no tiene opciones de tratamiento al momento del diagnóstico ${ }^{6}$. El enfermo terminal tiene un pronóstico fatal en un plazo breve, descrito como menor a 6 meses $^{7}$. Esto debe ser determinado en forma precisa por un médico experto.

Tradicionalmente, la nomenclatura internacional ha dividido el concepto de Eutanasia en activa y pasiva. Sin embargo, en los últimos 15 años hay organismos y autores nacionales que plantean esta distinción como inapropiada, postulando que la eutanasia es siempre una acción activa $^{8-10}$. Para efectos de la discusión, dividiremos conceptualmente los dos tipos de eutanasia.

\section{Eutanasia activa}

El acto médico de terminar con la vida de un paciente, bajo la voluntad del mismo paciente ${ }^{11}$. Esto, bajo las condiciones previamente descritas, $\mathrm{y}$ al alero de una normativa que lo regularice.

\section{Eutanasia pasiva}

El acto médico de retirar, suspender o no iniciar un tratamiento que se limita a prolongar la vida de un paciente que se encuentra en situación de enfermedad terminal o irreversible ${ }^{12,13}$.

\section{Limitación del esfuerzo terapéutico (LET)}

Consiste en no aplicar medidas desproporcionadas para propósitos terapéuticos en pacientes con mal pronóstico ${ }^{14}$. Contempla tanto la omisión como el retiro de tratamientos.

La distinción entre LET y Eutanasia pasiva- permanece en discusión por parte de los expertos. Para efectos de esta revisión, adheriremos a los autores que plantean eutanasia pasiva y LET como sinónimos ${ }^{13}$.

\section{Directrices anticipadas/ avanzadas ${ }^{15}$}

Estos son documentos reconocidos legalmente cuyo propósito es definir anticipadamente el cuidado médico que desea el paciente en el caso de quedar incapacitado para tomar decisiones. Este es valorado como una extensión del principio de autonomía ${ }^{16}$.

Efectivamente las directrices han demostrado asociarse a resultados positivos en torno al respeto de la autonomía del paciente. Estudios han mostrado que las personas que dejaban directrices anticipadas tenían más tendencia a fallecer fuera del hospital, así como a niveles significativamente menores de gastos en salud ${ }^{17,18}$. También se ha mostrado que más de la mitad de los pacientes en estado terminal rechazan medidas artificiales de nutrición ${ }^{19}$.

Es necesario resaltar un estudio que concluye que a la hora de decidir privar de alimentación y fluidos a un grupo de pacientes terminales con demencia, el equipo médico privilegia su propia evaluación de la condición médica de los pacientes y la opinión de los familiares por sobre las directrices anticipadas ${ }^{20}$. Es decir, la existencia de directrices anticipadas no asegura el cumplimiento de las mismas, y estas se deben someter a evaluación constante para promover su cumplimiento.

\section{Situación internacional}

\section{1) Eutanasia activa}

Actualmente, hay 4 países en el mundo (Holanda, Bélgica, Luxemburgo, Colombia) en los cuales la eutanasia activa es legal. La situación en cada uno de estos países, será motivo de otra revisión.

\section{2) Eutanasia pasiva}

Hay numerosos países que la permiten formalmente a través de sus regulaciones en diferentes formas, como lo son Canadá, España, Inglaterra, India, entre otros. A pesar de las legislaciones que la niegan (como la chilena), es una práctica ampliamente usada en Medicina Intensiva en todo el mundo. 


\section{Decisión de no reanimar}

Un ejemplo específico de regulación de eutanasia pasiva, a través de una directriz anticipada, es la orden "Do not attemp resucitation (DNAR)", documento que puede firmar cualquier ciudadano del Reino Unido para constatar que no desea reanimación cardiopulmonar en caso de paro cardiorrespiratorio $^{21}$.

\section{Decisión de no escalar en tratamientos complejos}

Hay una serie de procedimientos médicos de variable complejidad que se pueden limitar. Entre estos se encuentran la Ventilación mecánica invasiva, cateterismo central, intervenciones quirúrgicas, alimentación e hidratación, entre otros.

A modo de ejemplo, en España, desde el año 2000 entra el vigor el Convenio para protección de los derechos humanos, en el cual se contemplan las directrices anticipadas para la regulación de eutanasia pasiva ${ }^{22}$. En la Tabla 1 , se ejemplifica un extracto de formato tipo de directriz anticipada de la comunidad autónoma de Madrid ${ }^{23}$.

En la Tabla 2, se presenta un resumen sobre qué aceptan las legislaciones actuales en cuanto a eutanasia pasiva en algunos países (información pública a través de sitios electrónicos oficiales de organismos gubernamentales).

\section{Legislación chilena actual}

En Chile, actualmente la eutanasia se excluye en la Ley de Derechos y Deberes del Paciente, promulgada el año 2012. En el artículo 16 de ésta, se manifiesta "La persona que fuere informada de que su estado de salud es terminal, tiene derecho a otorgar o denegar su voluntad para someterse a cualquier tratamiento que tenga como efecto prolongar artificialmente su vida, sin perjuicio de mantener las medidas de soporte ordinario. En ningún caso, el rechazo de tratamiento podrá implicar como objetivo la aceleración artificial del proceso de muerte" 24 .

También en el artículo 14, la misma ley refiere "En ningún caso el rechazo a tratamientos podrá tener como objetivo la aceleración artificial de la muerte, la realización de prácticas eutanásicas o el auxilio al suicidio".

En el año 2014 se abrió el debate en torno a la eutanasia en Chile debido al caso del Dr. Manuel Almeyda, ex académico de la Universidad de Chile, quien sufría una insuficiencia respiratoria y cardiaca, haciéndolo oxígeno dependiente y provocándole una ingrata calidad de vida. En enero de 2014, escribió una carta al Presidente del Colegio Médico solicitando eutanasia. Frente a la negación a su solicitud, murió poco tiempo después tras negarse a comer por 1 semana, con apoyo de sus familiares.

En respuesta a esta situación, el 2014 se ingresa una solicitud de proyecto de Ley a la comisión de salud para legislar sobre eutanasia. Ésta se actualizó y reingresó posteriormente en respuesta al caso de Valentina Maureira, adolescente portadora de fibrosis quística avanzada, quien en un vídeo se dirige a la presidenta de la República, solicitando eutanasia. La idea de discutir el tema ha sido rechazada retiradamente por la Comisión de Salud del Senado.

\section{Tabla 1. Formato de Directriz anticipada Comunidad Autónoma de Madrid (extracto) ${ }^{33}$}

Instrucciones que quiero que se tengan en cuenta en mi atención médica:

Deseo finalizar mi vida sin la aplicación de técnicas de soporte vital, respiración asistida o cualquier otra medida extraordinaria, desproporcionada y fútil que sólo esté dirigida a prolongar mi supervivencia artificialmente, o que estas medidas se retiren, si ya han comenzado a aplicarse

Deseo que se me proporcionen los tratamientos necesarios para paliar el dolor físico o psíquico o cualquier síntoma que me produzca una angustia intensa

Rechazo recibir medicamentos o tratamientos complementarios y que se me realicen pruebas o procedimientos diagnósticos, si en nada van a mejorar mi recuperación o aliviar mis síntomas

Deseo que se facilite a mis seres queridos y familiares el acompañamiento en el trance final de mi vida, si ellos así lo manifiestan y dentro de las posibilidades del contexto asistencial

Deseo me sean aplicados todos los tratamientos precisos para el mantenimiento de la vida hasta donde sea posible, según el buen criterio médico 
Tabla 2. Situación sobre aspectos de la eutanasia pasiva en algunos países

\begin{tabular}{|lccccc|}
\hline País & $\begin{array}{c}\text { Decisión de } \\
\text { no escalar en } \\
\text { tratamientos } \\
\text { complejos }\end{array}$ & $\begin{array}{c}\text { Decisión } \\
\text { de no } \\
\text { reanimar }\end{array}$ & $\begin{array}{c}\text { Directrices } \\
\text { anticipadas }\end{array}$ & $\begin{array}{c}\text { Suspender } \\
\text { cuidados que } \\
\text { mantienen la } \\
\text { vida }\end{array}$ & Comentarios \\
\hline Reino Unido & $X$ & $X$ & $X$ & $\begin{array}{c}\text { Según ley, todos los adultos con capaci- } \\
\text { dad de decidir tienen la posibilidad de } \\
\text { rechazar tratamiento médico }\end{array}$ \\
\hline España & $X$ & $X$ & $X$ & $X$ & El 2008 se legisló en México la "Ley \\
México & $X$ & $X$ & $X$ & $\begin{array}{l}\text { de Voluntad Anticipada", creando un } \\
\text { organismo gubernamental Ilamado } \\
\text { "Coordinación de voluntades anticipa- } \\
\text { das", adscrito a la Secretaría de Salud } \\
\text { del Estado de México, que regula las } \\
\text { directrices anticipadas }\end{array}$ \\
\hline Canadá & $X$ & & $X$ & $\begin{array}{l}\text { La Corte Suprema de Canadá, ha acor- } \\
\text { dado para Junio del 2016 legalizar la } \\
\text { eutanasia activa }\end{array}$ \\
\hline
\end{tabular}

X: Las personas en ese país pueden optar a estas formas de eutanasia pasiva.

\section{La opinión de la ciudadanía}

En diversos catastros o estudios de opinión, ha quedado claro que la mayoría de la población chilena apoya la eutanasia.

En los resultados de la Encuesta Bicentenario de la Universidad Católica, 52\% de los chilenos y chilenas está de acuerdo con que "terminar la vida de los enfermos incurables (eutanasia) es un acto justificable bajo ciertas circunstancias". Un dato interesante es que la misma encuesta señala que el, acuerdo que es de $64 \%$ en el estrato alto, $52 \%$ en el medio y $48 \%$ en el bajo.

Luego, en la encuesta de la Universidad Diego Portales, también se ha preguntado sobre el tema. En el 2014, 68\% de las personas está de acuerdo con la muerte asistida, con $27 \%$ en contra. En las 3 aplicaciones anteriores de la encuesta $(2010,2012,2013)$, se ha mostrado una clara predominancia en la respuesta "Estoy muy de acuerdo con la afirmación: Un enfermo terminal tiene derecho a solicitar su muerte asistida-eutanasia".

Estos sondeos de opinión evidencian que la ciudadanía hoy avala el apoyo a la regulación del final de la vida y que es necesario abrir la discusión a nuestra sociedad.
Discusión: eutanasia pasiva y activa en Chile

"Nada sobre mí, sin mi” Benedetto Saraceno ${ }^{25}$

En la revisión sobre los tratados internacionales sobre derechos y derechos humanos de la ONU, OMS y la Convención Americana de Derechos Humanos, entre otras, destaca que, si bien la vida se menciona en múltiples artículos como derecho fundamental, hay una completa ausencia de artículos que traten el derecho a morir sin sufrimiento y respetando la autonomía del paciente.

En función de estos derechos mencionados, planteamos la necesidad de morir dignamente, como un derecho humano fundamental. Creemos que el derecho a vivir como deseemos se puede ampliar a la posibilidad de decidir cómo queremos morir, respetando la voluntad de las personas y su dignidad.

El modelo asistencial "centrado en el paciente" desarrollado en los últimos 30 años, pone el foco en estructurar los sistemas de salud en respuesta a los valores y preferencias de la población. Debe primar la auto-determinación de los sujetos, condición sine qua non para la acción médica. Transgredir este principio significa violentar derechos. 


\section{Eutanasia pasiva}

En la "Convención interamericana sobre la protección de los derechos humanos de las personas mayores" generada por la Organización de los Estados Americanos, actualmente en discusión en los parlamentos nacionales para ratificación, se plantea el rol de los estados en que “ Las instituciones públicas o privadas y los profesionales de la salud no podrán administrar ningún tratamiento, intervención o investigación de carácter médico o quirúrgico, sin el consentimiento informado de la persona mayor"26.

Consideramos que la legislación chilena actual es poco precisa con respecto a la regulación de la eutanasia pasiva y limitación del esfuerzo terapéutico. Pese a que sabemos que en medicina intensiva estas son prácticas comunes en nuestro país, la legislación confunde, ya que: 1) No define prácticas eutanásicas; 2) Al plantear que "en ningún caso el retiro de tratamientos puede acelerar artificialmente el proceso de muerte" da para una amplia interpretación, ya que podríamos pensar que el retiro, por ejemplo, de ventilación mecánica podría acelerar la muerte.

Por otro lado, es importante reconocer que en nuestro país se promueve escasamente la autonomía de las personas. Los médicos suelen entregar la información de manera inadecuada y no involucran a los pacientes en las decisiones que toman. El hecho de que la eutanasia pasiva no esté claramente normada en la legislación, deja a total discreción del médico cuanto involucrar al paciente, dañando profundamente su autonomía.

Por lo tanto, es necesario avanzar a una legislación que acepte y norme la eutanasia pasiva o limitación del esfuerzo terapéutico como práctica, ya que esto evitaría, por un lado, confusiones innecesarias y por otro, podría mejorar la autonomía de los pacientes. Junto con las regulaciones de esta legislación, debemos avanzar en generar un Formato de Directriz Anticipada, de fácil acceso y con educación amplia a la población para su implementación.

\section{Eutanasia activa}

El foco de esta discusión ciudadana debe estar centrado en la autonomía de los seres humanos para decidir qué hacer con su cuerpo, particularmente en momentos de gran sufrimiento.

Sobre el sufrimiento, reconocemos diversos tipos: físico, emocional y espiritual. Con respecto al físico, es prioritario desarrollar una política de cuidados paliativos a nivel nacional de la mano con la legislación sobre eutanasia ${ }^{27}$.

Sin embargo, aun cuando alcanzáramos cuidados paliativos integrales, debemos reconocer que la analgesia tiene limitaciones. Se ha descrito que, además del conocido efecto de tolerancia que producen los opioides, hay $10 \%$ de la población que no responde a morfina. Se suma a esto los efectos secundarios de la analgesia, más frecuentes en los adultos mayores ${ }^{28}$. Por otro lado, es importante destacar los otros tipos de sufrimiento, evitando reducir al enfermo sólo a los síntomas directos de su enfermedad. Cuando vemos a una persona al final de la vida, su sufrimiento no tiene que ver exclusivamente con sus dolencias físicas, sino que muchas veces trasciende a lo emocional y espiritual, dolencias mucho más complejas y difíciles de abordar. Reconocer este padecimiento nos lleva a plantear el derecho que tienen las personas en estado terminal a decidir cuándo desean poner fin a dicho dolor.

En relación a la autonomía, como ya ha sido planteado, esta se respeta sólo en una fracción de los pacientes en etapa paliativa. Creemos firmemente que el principio ético de la autonomía es lo que debe imperar en los pacientes terminales. Al tener menos de 6 meses de pronóstico de vida, como sociedad debemos concederles la posibilidad de tomar la elección de cómo y cuándo desean morir.

Por otro lado, es importante abarcar el concepto de dignidad. Es osado hablar de muerte digna por su carácter subjetivo, pero sabemos que los propios usuarios sienten pérdida de dignidad cuando no son respetados en su decisión personal ${ }^{29}$. Hay diferencias culturales con respecto a los cuidados al final de la vida que son significativos, por lo que no es posible homogeneizar los cuidados para toda la población ${ }^{30}$. La dignidad es subjetiva e individual, por lo que el sistema de salud debe adaptarse a los requerimientos culturales y personales de cada individuo para acompañarlos en una partida digna.

Con respecto a este punto, es necesario añadir a la discusión, las tremendas inequidades que sufre nuestra población al final de la vida. Chile es uno de los países con mayor desigualdad económica en el mundo. Estas diferencias impiden que las personas de estratos bajos puedan morir con dignidad. La desigualdad en el acceso de los 
pacientes a cuidados paliativos de enfermedades crónicas o catastróficas genera que algunos no tengan las condiciones suficientes para afrontar de una manera digna y moderna sus enfermedades.

Por lo tanto, un paso necesario y paralelo a la eutanasia es normar los cuidados paliativos para que efectivamente sean aplicados a todos los enfermos terminales que lo requieran.

\section{Consideraciones finales}

Ante la falta de una discusión clara y abierta acerca de los dilemas de la muerte que se dan cita en las profesiones de salud y, en particular, de la eutanasia, esta revisión pretende ser un aporte a impulsar un mejor debate en torno al tema que, aunque en las sombras, afecta diariamente a parte de nuestros pacientes.

Y es que la falta de articulación de nuestra legislación y nuestras prácticas clínicas, termina vulnerando sistemáticamente el derecho a la autodeterminación sobre la propia vida a las personas enfermas. En ese sentido, es preciso constatar que lo que entendemos por vivir bien, es algo que no tiene un contenido predeterminado sino que está sujeto a discusiones colectivas y también a determinaciones personales. Tenemos derecho a reivindicar la autonomía sobre nuestras vidas en la medida que es parte integral de la dignidad humana y a la vez es parte de las formas de expresión de ésta.

Como sociedad debemos definir hasta dónde es prudente llegar en la ayuda a una persona que está sufriendo con una enfermedad terminal, sabiendo que los cuidados paliativos, como los opioides o la morfina, ya no serán suficientes para evitar el sufrimiento.

Si bien en los catastros citados se ve una mayoría de la población de acuerdo con la eutanasia, el tema en Chile sigue estando oculto, incluso dentro del ámbito de las discusiones de profesionales sanitarios. Identificamos un vacío en la legislación actual. En la práctica, la toma de decisiones se dificulta por la falta de un marco legal claro. A esto se suma la asimetría de información que existe con los pacientes, sin vías claras sobre cómo ejercer sus derechos.

En la práctica, lo que acaba ocurriendo al aplicar la legislación vigente, sin dar espacio a la reflexión crítica acerca de ella, es que lo que pre- domina es una imposición cultural de las creencias religiosas de un grupo a toda la sociedad, pese a que el Estado se proclama laico y respetuoso de la libertad personal.

Por lo tanto, una tarea fundamental que es posible desprender del análisis previo es la de ampliar el debate en Chile, reflexionar en el seno de las organizaciones académicas, sociales y las familias sobre el tema, informando a la ciudadanía sobre los componentes e implicancias de la eutanasia, en función de tener como sociedad entera una discusión abierta, clara y transparente.

\section{Referencias}

1. Bilansky A. Impacto de la tecno-ciencia en el afrontamiento de la enfermedad y la muerte. Rev Geriatría Clínica Web 2010; 4 (3): 0000-0000.

2. Taboada P. El derecho a morir con dignidad. Acta Bioeth 2000; 6 (1): 89-101.

3. Rodríguez R. El derecho a amar y el derecho a morir: entre la vida y la muerte. Fondo Editorial PUCP; 1997. $364 \mathrm{p}$.

4. Mannará F. Concepción de muerte en las culturas oriental y occidental. Rev Geriatría Clínica Web 2010; 4 (3): 0000-0000.

5. Ping-cheung L. Euthanasia and Assisted Suicide from Confucian Moral Perspectives. Dao 2010; 9 (1): 53-77.

6. El enfermo terminal. Rev Med Chile 2000; 128 (5): 54752.

7. Quill TE. Initiating end-of-life discussions with seriously ill patients: Addressing the 'elephant in the room'. JAMA J Am Med Assoc 2000; 284 (19): 2502-7.

8. Materstvedt LJ, Clark D, Ellershaw J, Førde R, Gravgaard A-MB, Müller-Busch HC, et al. Euthanasia and physician-assisted suicide: a view from an EAPC Ethics Task Force. Palliat Med 2003; 17 (2): 97-101-79.

9. Goic GA. Apuntes sobre la eutanasia. Rev Med Chile 2005; 133 (3): 371-5.

10. Beca J, Ortiz A, Solar S. Derecho a morir: un debate actual. Rev Med Chile 2005; 133: 601-6.

11. de Wachter MM. Active euthanasia in the netherlands. JAMA 1989; 262 (23): 3316-9.

12. Garrard E, Wilkinson S. Passive euthanasia. J Med Ethics 2005; 31 (2): 64-8.

13. Simón Lorda P, Barrio Cantalejo IM, Alarcos Martínez FJ, Barbero Gutiérrez J, Couceiro A, Hernando Robles P. Ética y muerte digna: propuesta de consenso sobre un uso correcto de las palabras. Rev Calid Asist 2008; 23 (6): 271-85. 
14. Herreros B, Palacios G, Pacho E. Limitation of the therapeutic effort. Rev Clínica Esp 2012; 212 (3): 134-40.

15. Otte IC, Elger B, Jung C, Bally KW. The utility of standardized advance directives: the general practitioners' perspective. Med Health Care Philos. 2016.

16. Winzelberg GS, Hanson LC, Tulsky JA. Beyond autonomy: diversifying end-of-life decision-making approaches to serve patients and families. J Am Geriatr Soc 2005; 53 (6): 1046-50.

17. Degenholtz HB, Rhee Y, Arnold RM. Brief Communication: The Relationship between Having a Living Will and Dying in Place. Ann Intern Med 2004; 141 (2): 1137.

18. Nicholas LH, Langa KM, Iwashyna TJ, Weir DR. Regional Variation in the Association Between Advance Directives and End-of-Life Medicare Expenditures. JAMA 2011; 306 (13): 1447-53.

19. Martínez Almazán E, AltadillArdit A, Navarro GAJ. Disposiciones previas: experiencia piloto en una residencia de ancianos. Rev Esp Geriatría Gerontol 2002; 37 (4): 203-9.

20. The A, Pasman R, Onwuteaka-Philipsen B, Ribbe M. Withholding the artificial administration of fluids and food from elderly patients with dementia: ethnographic study. BMJ 2002; 325 (7376): 1326.

21. Stewart Kevin, Spice Claire, Rai G. Where now with Do Not Attempt Resuscitation decisions? Age Ageing 2003; 32 (2): 143-8.
22. Martínez K. Los documentos de voluntades anticipadas. An Sist Sanit Navar 2007; 30: 87-102.

23. Derecho a morir dignamente. El testamento vital en la Comunidad de Madrid (Documento de instrucciones previas) [Internet]. 2012 [citado el 23 de febrero de 2016]. Available from:www.cruzrojamadrid.org.

24. Ministerio de Salud (Chile). Regulo los derechos y deberes que tienen las personas en relación con acciones vinculadas a su atención en salud. Biblioteca del Congreso Nacional; 2012.

25. Saraceno Benedetto. Liberación de los pacientes psiquiátricos, La. Editorial Pax México; 2003. 164 p.

26. Consejo permanente de la organización de los estados americanos. Proyecto de resolución: Convención interamericana sobre la protección de los derechos humanos de las personas mayores. 2015.

27. World Health Organization. Better Palliative Care for Older People [Internet]. World Health Organization; 2004. Available from: www.euro.who.int

28. Wilder-Smith OHG. Opioid use in the elderly. Eur J Pain 2005; 9 (2): 137-40.

29. Chochinov HM, Hack T, Hassard T, Kristjanson LJ, McClement S, Harlos M. Dignity in the terminally ill: a cross-sectional, cohort study. Lancet Lond Engl 2002; 360 (9350): 2026-30.

30. Doolen J, York N. Cultural Differences With End-of-Life Care in the Critical Care Unit. Dimens Crit Care Nurs 2007; 26 (5): 194-8. 